\title{
Electrophysiology in a district general hospital
}

\author{
Atul Prakash, Phyllis M Holt
}

\begin{abstract}
Objective-To investigate the feasibility of performing electrophysiological studies at a district general hospital and to evaluate the importance of such studies in the management of patients with suspected arrhythmias.
\end{abstract}

Design-Retrospective study of patients having had electrophysiological studies during a three year period.

Setting-District general hospital.

Subjects-93 patients (50 men, 43 women, mean age 45.9 years) with suspected arrhythmias.

Results-The patients were divided into two groups according to symptoms. Group 1 (34 patients) presented with syncope. Group 2 (59 patients) presented with palpitation. All had previously undergone non-invasive investigations. All had had multiple hospital admissions and outpatient attendances. In group 1 nine patients with no documented arrhythmias had inducible ventricular tachycardia and three of six with suspected bradyarrhythmias had ventricular tachycardia. Fourteen patients had suspected ventricular arrhythmias before electrophysiological studies, which were confirmed in all, four receiving automatic implantable cardioverter defibrillators. Electrophysiological studies were used to guide drug treatment in all patients. Group 2 consisted of 32 patients with reentrant supraventricular tachycardia and 15 with ventricular tachycardia; 12 had no documented arrhythmias. In those with supraventricular tachycardia, accessory pathways were identified in all. In 23 patients drug treatment (guided by electrophysiological studies) was successful. In nine, drug treatment guided by electrophysiological studies were ineffective and radiofrequency ablation was successful. In 15 patients with ventricular tachycardia and palpitations, 10 had their drugs changed after electrophysiological studies and their ventricular tachycardia was suppressed. In five patients electrophysiological studies showed that ventricular tachycardia was unsuppressed and they were referred for an operation or implantation of an automatic cardioverter defibrillator. In 12 patients with no documented arrhythmias electrophysiological studies identified significant arrhythmias in six. There were no complications.

Conclusions-Diagnostic electrophysiological studies can safely and effectively be performed in a district general hospital. These studies are especially effective in investigating patients with syncope, and also provide a strategy for future arrhythmia management.

(Br Heart $\mathcal{F} 1995 ; 73: 76-81)$

Keywords: electrophysiology, district general hospital.

Clinical electrophysiology is important in the diagnosis of patients with arrhythmias, and vital in providing a management strategy for them. The success of radiofrequency ablation in patients with accessory pathways ${ }^{1-3}$ and the identification of patients at high risk with ventricular arrhythmias ${ }^{45}$ increases the need for electrophysiological studies still further.

At present such studies are performed almost exclusively at regional centres. Their medical, technical, and laboratory resources will be increasingly taken by the time consuming therapeutic ablation procedures, thereby further extending waiting list times for those in need of routine diagnostic studies.

Our objectives were twofold. Firstly, to show that diagnostic electrophysiological studies can be performed safely and effectively at a district general hospital and, secondly, that such studies significantly modified and improved the future management of those patients. We hope that this will add weight to the thesis that electrophysiological studies are not a "last resort" in the management of patients with proved or suspected arrhythmias, ${ }^{67}$ but are, in fact, an important tool whose early use leads to considerable clinical and financial benefits.

\section{Patients and methods}

In total, 93 patients underwent electrophysiological studies at Maidstone District General Hospital during a three year period from May 1990 to April 1993. There were 50 men and 43 women aged 15 to 80 years (mean 45.9 years). Eighty patients were referred from Maidstone district and 13 from neighbouring districts for investigation of suspected or proved arrhythmias. Therefore in the three years of the study 80 patients had 
electrophysiological studies from Maidstone district (population 200000 ) - that is, $120 / 10^{6}$ each year. Demographically, Maidstone district has an age distribution and an incidence of ischaemic heart disease comparable with the national average.

All patients had previously undergone noninvasive investigations, including 12 lead electrocardiography, echocardiography, Holter monitoring, and exercise testing. All patients had had two to five outpatient visits (mean 3.5 visits) and most had had previous emergency admissions (none to three; mean 0.92 admissions). Electrophysiological studies were undertaken for the following reasons: $(a)$ in patients with symptoms of palpitation or syncope strongly suspected to be secondary to arrhythmia, which had not been proved noninvasively; (b) to optimise medical treatment in patients with arrhythmias previously refractory to empirical drug treatment; and (c) to clarify future arrhythmia management strategy and facilitate interventions (for example, radiofrequency ablation or automatic implantable cardioverter defibrillator implantation at a tertiary centre).

In group 1, 25 patients were subsequently documented to have underlying cardiac disease, 20 with ischaemic heart disease and five with congestive cardiomyopathy. In group 2, 19 patients had underlying heart disease, 16 with ischaemic heart disease and three with congestive cardiomyopathy. Underlying cardiac disease was found predominantly in the patients with known ventricular tachycardia (10 with ischaemic heart disease, three with congestive cardiomyopathy) and the group with atrial fibrillation (four with ischaemic heart disease).

\section{METHODS}

Most patients were studied as day cases. Those with ventricular tachyarrhythmias required a longer period of hospital admission. The patients were fasted, premedicated, and bipolar 6 French USCI leads were inserted via the right femoral vein after local anaesthesia with $1 \%$ lignocaine. Three leads were inserted and positioned initially at the high right atrium, bundle of His, and right ventricular apex. Leads were subsequently manipulated to other sites-for example, coronary sinus or right ventricular outflow or inflow as required during the studies. Signals were recorded on a six channel Picker $\mathrm{KD}$ 6000 machine with three simultaneous electrocardiogram leads and three simultaneous intracardiac signals displayed. Filtering was between 50 and $500 \mathrm{~Hz}$. Intracardiac stimulation was performed using a Biotronik VH 10 electrophysiological stimulator.

To establish electropharmacological efficacy, intravenous antiarrhythmic drugs were administered according to a set protocol ${ }^{8}$ and repeat stimulation studies undertaken. The response to intravenous antiarrhythmic drugs was used to indicate long term drug treatment by mouth.

In patients with ventricular tachycardias the drugs tested were disopyramide, mexiletene, sotalol (where left ventricular function allowed), and amiodarone. In those in whom the ventricular tachycardia was stable and well tolerated, the response was assessed by intravenous administration of the drug chosen during the tachycardia. No more than one drug was administered intravenously in each study. Where the arrhythmia was unstable or not well tolerated, and also for pre-discharge assessment, the effectiveness of the antiarrhythmic drug given by mouth was assessed when adequate plasma concentrations were achieved. In patients with syncope or known ventricular arrhythmias, all 12 electrocardiogram leads were attached during the electrophysiological studies. For all studies full facilities for cardiopulmonary resuscitation were available.

The studies were performed by a single cardiologist (PH) with previous electrophysiology training, or a cardiac registrar (AP) under supervision. An average of one to two electrophysiological studies were performed each week.

Patients requiring therapeutic intervention were referred to a regional centre with a full report and copies of the relevant results.

\section{Results}

Ninety three patients were studied (50 men, 43 women, mean age 45.9 years). The procedure times ranged from 45 to 90 minutes (mean 65 minutes) with a screening time of five to 15 minutes (mean 13 minutes). No complications were encountered. Follow up ranged from three to 36 months.

Outpatient follow up was undertaken at two, six, and 12 months after the electrophysiological studies. Patients with recurrent arrhythmias were reviewed more often, as necessary.

The results are considered in two sections on the basis of presenting symptoms.

\section{GROUP 1: PATIENTS PRESENTING WITH SYNCOPE}

There were 34 patients (mean age 49.8 years), 14 with no arrhythmias on non-invasive investigation, and six with bradycardias as the presumed aetiology for their syncope (two with bifasicular block on 12 lead electrocardiology and four with non-diagnostic episodes of sinus bradycardia on Holter monitoring). The remaining 14 patients in group 1 had presumed ventricular arrhythmias and evidence of sustained or non-sustained ventricular tachycardia on Holter monitoring, which was not associated with syncope, their presenting symptom.

Electrophysiological testing significantly influenced the diagnosis in those patients with syncope and no documented arrhythmia, and in those with presumed bradyarrhythmias. Nine of the 14 patients with no previously documented arrhythmias had sustained ventricular tachycardia induced and three of six patients with presumed bradycardia had in fact ventricular tachycardia as the basis of their syncope (figs 1 and 2). Table 1 summarises the results for group 1 patients. 


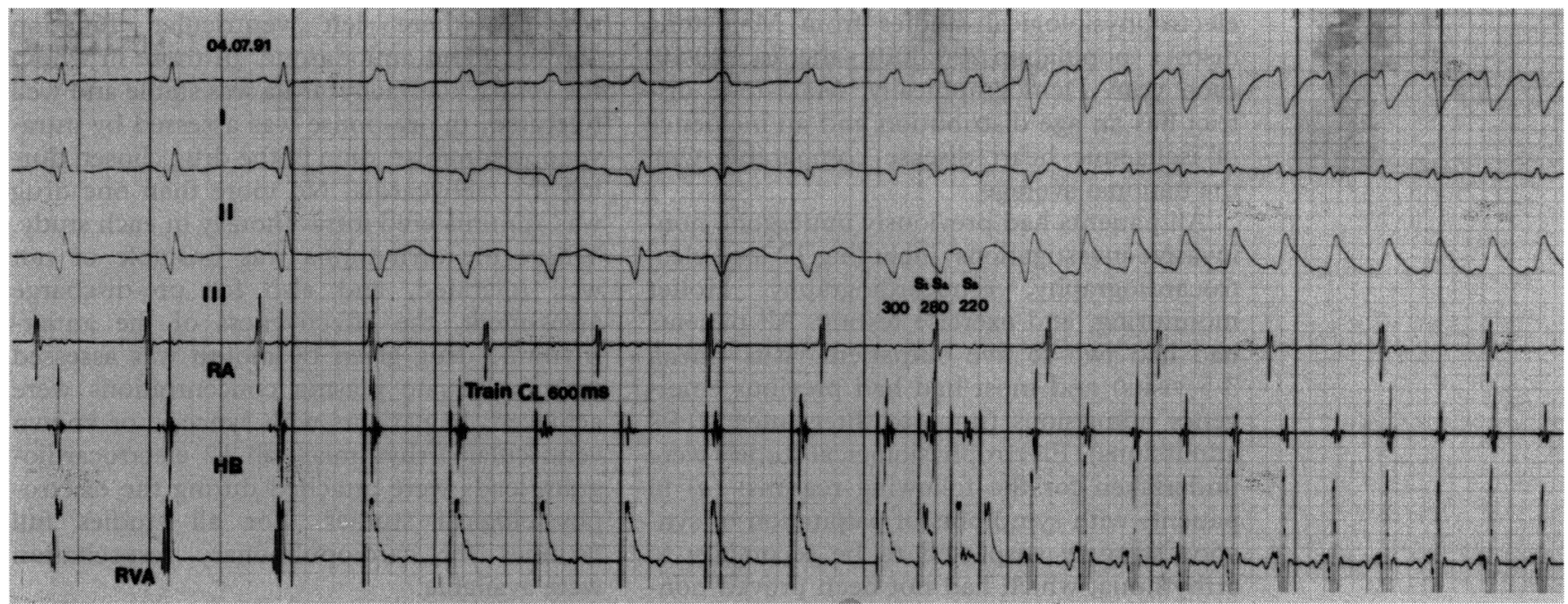

Figure 1 Patient with right bundle branch block and inducible sustained monomorphic ventricular tachycardia on programmed stimulation. RA $=$ Right atrial electrogram; $H B=H$ is bundle electrogram; and $R V A=$ electrogram from right ventricular apex.

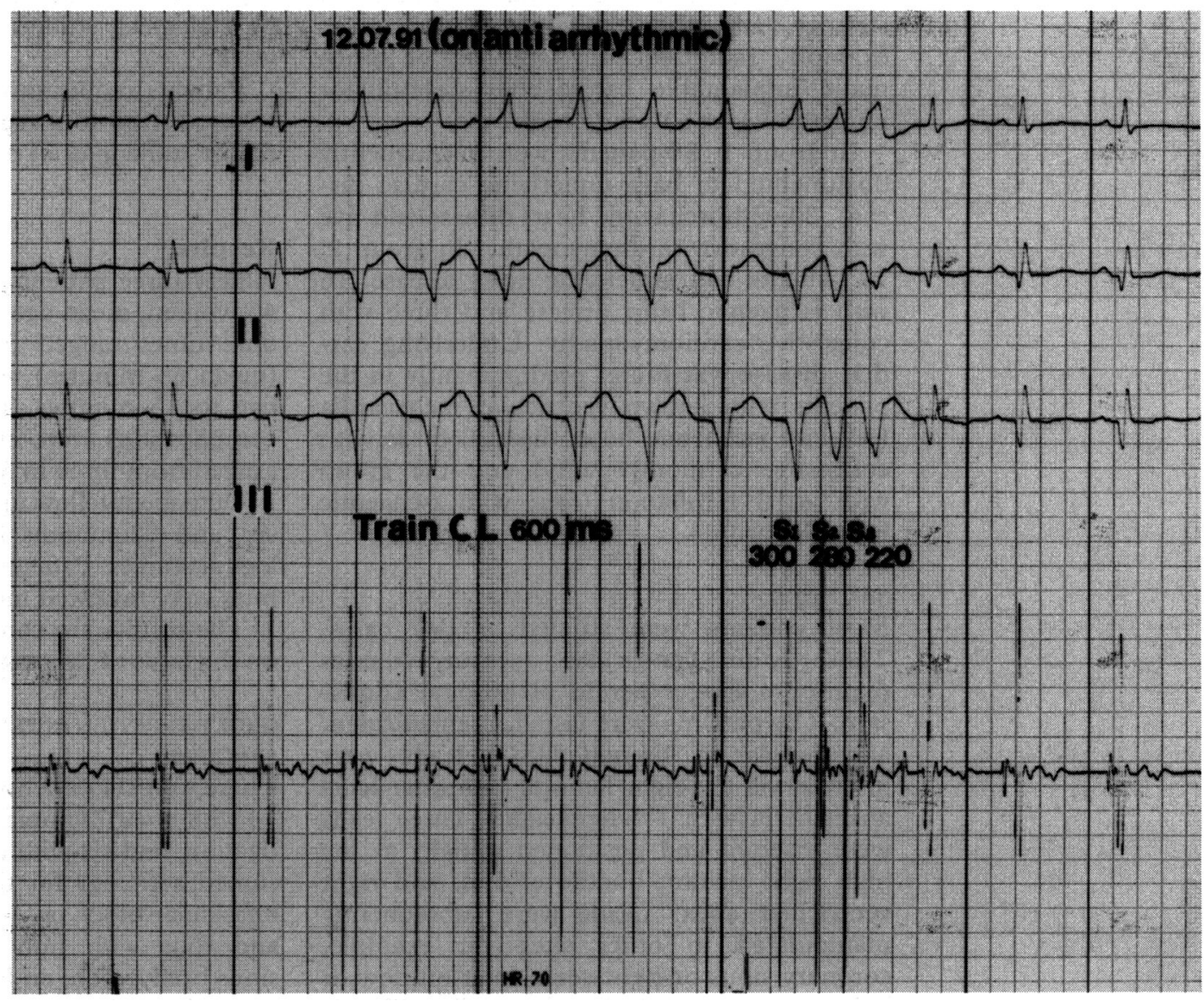

Figure 2 Same patient as in fig 1. Repeat programmed stimulation after six weeks of amiodarone treatment shows ventricular tachycardia can no longer be induced (abbreviations as in fig 1).

Table 1 Group 1: Patients with recurrent syncope

\begin{tabular}{|c|c|c|c|}
\hline Pre-EPS & EPS & Outcome after EPS & $\begin{array}{l}\text { Long term follow up and } \\
\text { management }\end{array}$ \\
\hline $\begin{array}{l}14 \text { patients. } \\
\text { No arrhythmia documented }\end{array}$ & $\begin{array}{l}\text { VT }(n=9) \\
\text { Non-sustained VI: }(n=1) \\
\text { Normal study }(n=4)\end{array}$ & $\begin{array}{l}\text { Guided drugs successful }(n=7) \\
\text { Guided drugs unsuccessful }(n=2) \\
\text { Abnormal BEG }(n=2)\end{array}$ & $\begin{array}{l}\text { Well. No hospital admissions } \\
\text { AICD, alive } \\
\text { Well. Receiving } \\
\text { anticonvulsant drugs }\end{array}$ \\
\hline Six patients presumed bradycardia & $\begin{array}{l}\operatorname{VTT}(n=3) \\
\text { Confirmed SSS }(n=3)\end{array}$ & $\begin{array}{l}\text { Guided drugs successful } \\
\text { Atrial pacemakers }\end{array}$ & $\begin{array}{l}\text { Well. Receiving drugs } \\
\text { Well }\end{array}$ \\
\hline 14 patients presumed VT & $\begin{array}{l}\text { Inducible VF }(n=2) \\
\text { Inducible VT }(n=12)\end{array}$ & $\begin{array}{l}\text { AICD } \\
\text { Guided drugs successful }(n=9) \\
\text { VT inducible despite drugs }(n=3)\end{array}$ & $\begin{array}{l}\text { Alive and well } \\
\text { Alive and well } \\
\text { Alive with AICD }\end{array}$ \\
\hline
\end{tabular}

EPS = Electrophysiological studies; VT $=$ ventricular tachycardia; AICD $=$ automatic implantable cardioverter defibrillators; $\mathrm{EEG}=$ electroencephalogram; SSS = sick sinus syndrome; and VF = ventricular fibrillation. 
Figure 3 Patient with Wolff-Parkinson-White syndrome with re-entrant supraventricular tachycardia. The earliest retrograde atrial activation is seen in the electrogram recorded from the distal coronary sinus. Dcs = Electrogram recorded from the distal coronary sinus. $a$, atrial electrogram; $b$, His bundle electrogram; $v$, ventricular electrogram. Other abbreviations as in fig: 1 .

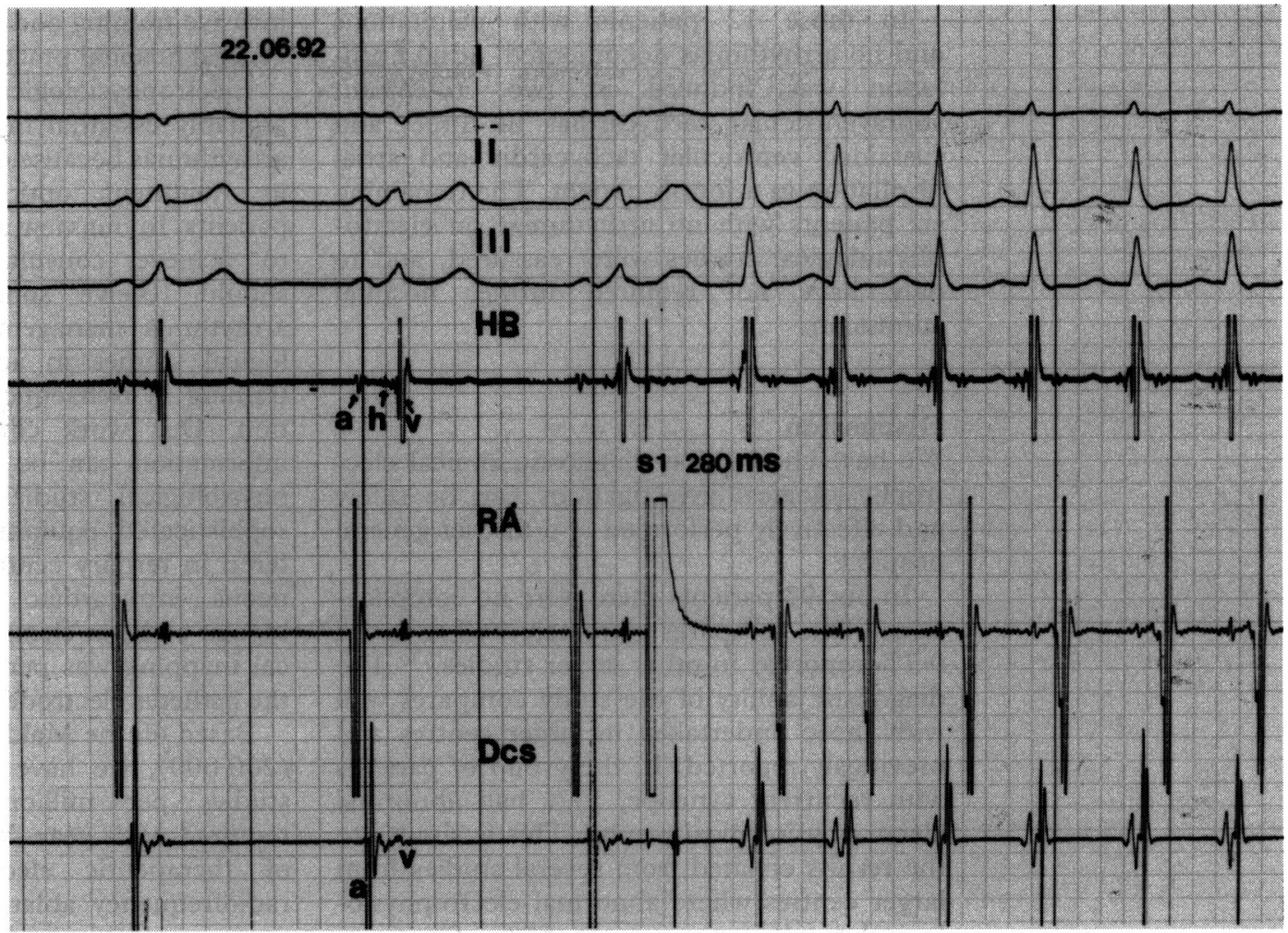

GROUP 2: PATIENTS PRESENTING WTTH PALPITATIONS

There were 59 patients presenting with palpitations; 32 patients had narrow complex re-entrant supraventricular tachycardias documented on Holter monitoring and 15 patients with palpitations had non-sustained ventricular tachycardia diagnosed on Holter monitoring (their arrhythmia on Holter monitoring did not coincide with their symptoms). All had previously been treated empirically and unsuccessfully and required multiple hospital admissions. Twelve patients with a history of palpitations were investigated with no documented arrhythmias on non-invasive investigation.

Electrophysiological study allowed pharma- cological investigation of antiarrhythmic treatment and (in those with re-entrant supraventricular tachycardia, documented the anatomical substrate for the arrhythmia (fig 3). Table 2 summarises the results of electrophysiological study in group 2 patients.

Intravenous antiarrhythmic drugs apparently successful at the time of electrophysiological studies were not necessarily successful long term when given by mouth. This was either because of patient intolerance or because of breakthrough arrhythmias. These patients were subsequently referred to the tertiary centre for the appropriate intervention already identified as the next option in the management strategy at the time of electrophysiological study.

Table 2 Group 2: Patients presenting with palpitations

\begin{tabular}{|c|c|c|c|}
\hline Pre-EPS & EPS & Outcome after EPS & $\begin{array}{l}\text { Long term follow up and } \\
\text { management }\end{array}$ \\
\hline $\begin{array}{l}32 \text { patients. } \\
\text { Known re-entrant SVT }\end{array}$ & $\begin{array}{l}13 \text { DAVNP } \\
19 \text { accessory AV pathway }\end{array}$ & $\begin{array}{l}\text { Guided drugs successful }(n=8) \\
\text { Guided drugs not successful }(n=5) \\
\text { Guided drugs successful }(n=15) \\
\text { Guided drugs ineffective }(n=4)\end{array}$ & $\begin{array}{l}\text { Remain well on drugs } \\
\text { RF ablation } \\
\text { Well. Receiving drugs } \\
(n=13) \\
\text { RF ablation }(n=2) \\
\text { RF ablation }\end{array}$ \\
\hline $\begin{array}{l}15 \text { patients. } \\
\text { Non-sustained VT on Holter } \\
\text { monitoring }\end{array}$ & Monomorphic VT & $\begin{array}{l}\text { VT non-inducible after guided } \\
\text { treatment }(n=10) \\
\text { VT inducible despite drugs }(n=5)\end{array}$ & $\begin{array}{l}\text { All alive } \\
\text { AICD }(n=1) \\
\text { VT RF ablation }(n=1) \\
\text { Well receiving same drugs } \\
(n=1) \\
\text { CABG }(n=1) \\
\text { Died awaiting AICD }(n=1)\end{array}$ \\
\hline $\begin{array}{l}12 \text { patients. } \\
\text { No arrhythmias documented }\end{array}$ & $\begin{array}{l}\text { Normal study }(n=6) \\
\text { AF }(n=2) \\
\text { Re-entrant SVT }(n=3) \\
\text { AF and VT }\end{array}$ & $\begin{array}{l}\text { Reassured } \\
\text { Drugs } \\
\text { Drugs } \\
\text { Drugs }\end{array}$ & Well \\
\hline
\end{tabular}

EPS = Electrophysiological studies; DAVNP = Dual atrial ventricular (AV) nodal pathway; CABG = coronary artery bypass orfing; RF = Radiofrequency ablation; AICD = automatic implantable cardioverter defibrillators; AF = atrial fibrillation; grafting; $R F=$ Radiofrequency ablation; $A$ AlCD = automatic implan
$\mathrm{VT}=$ ventricular tachycardia; and SVT = supraventricular tachycardia 
In those 12 patients with palpitations and no arrhythmias documented, atrial fibrillation was induced in two, re-entrant supraventricular tachycardias in three, and sustained ventricular tachycardia and atrial fibrillation in a fourth patient. The remaining six patients with no arrhythmias on electrophysiological studies were reassured, and to date have not required further hospital admission.

\section{Discussion}

We have shown that diagnostic clinical electrophysiological investigations can be safely and effectively performed at a district general hospital.

In our 93 patients there were no complications. This compares extremely well with the $0.7 \%$ reported in other larger studies. ${ }^{10}$ The diagnostic ability of the study compares well with those undertaken in larger centres and previously reported. In the group of patients with recurrent syncope, $57 \%$ had abnormal electrophysiological results. This is similar to the results collated from several studies from larger centres where abnormal electrophysiological results were seen in $55 \%$ of patients with syncope; the most common abnormality observed was ventricular tachycardia. ${ }^{11}$ Ventricular tachycardia has also been shown as the most often provoked abnormality in patients with syncope and bundle branch block. ${ }^{12}$ Fifty per cent of our patients with suspected bradyarrhythmia had inducible ventricular tachycardia.

Our results confirm that electrophysiological testing in patients with syncope or in patients with presumed bradycardias provides a high yield of previously unsuspected ventricular tachyarrhythmias. This is particularly so in patients with underlying heart disease, as in our study population.

Drug treatment chosen as a result of the electrophysiological study resulted in significantly better control of the patient's arrhythmias. Where further intervention was required, the results were utilised by the regional centre for radiofrequency ablation, or implantation of automatic implantable cardioverter defibrillators where appropriate. Full diagnostic studies were not repeated at the regional centre. Regional centre acceptance of electrophysiological studies performed at a district general hospital would depend on the links between the two hospitals and also on the quality of the studies. Electrophysiological testing at our centre was performed by a cardiologist trained in electrophysiology or by a registrar in training, under the supervision of the consultant cardiologist. Widespread devolution of electrophysiology studies from regional centres to district general hospitals would be restricted if no staff with adequate experience and training was available. The "boundaries" between district hospital and regional centre cardiologists are becoming increasingly blurred, however. Many cardiologists have appointments at district and tertiary centres and are able to bring invasive training and expertise to their district general hospital practice.

Electrophysiological studies are now arguably essential in patients with recurrent arrhythmias because of the significant increase in treatment options available to such patients. In our view all junior doctors wishing to achieve consultant cardiologist status should receive some formal training in arrhythmia management and electrophysiological studies in addition to conventional training in ischaemic heart disease intervention. Our work confirms that satisfactory information can be obtained from electrophysiological studies using relatively less sophisticated equipment than that encountered in tertiary centres. With three simultaneous intracardiac recordings and using bipolar catheter electrodes, adequate anatomical mapping was performed by repositioning the catheter electrodes.

Based on the Maidstone district population $(200000)$, we have shown that about 120 studies per million population may be required each year. With the greater success of therapeutic electrophysiology-that is, radiofrequency ablation - the need for diagnostic electrophysiology will probably increase.

In addition to the obvious clinical benefits of performing electrophysiological studies at a district general hospital, there could also be a financial benefit. The cost/benefit ratio of electrophysiologically guided drug treatment compared with an empirical approach has been shown to be 10:1 for patients with recurrent supraventricular arrhythmias and 18:1 for patients with recurrent ventricular arrhythmias. ${ }^{1314}$ An emergency admission in our hospital costs $£ 300$ for each 24 hours, compared with a day case electrophysiological procedure at $£ 250$. Before electrophysiological studies the mean number of inpatient admissions in this group of patients was $\mathbf{0 . 9 2}$. After electrophysiological study in a follow up period ranging from three months to three years, the number of emergency admissions was 0.46 for each patient.

These results confirm that electrophysiological studies should be undertaken early in the investigation of patients with syncope or palpitations, and that they can safely and successfully be performed at a district general hospital with adequately trained staff. This would reduce the pressure on electrophysiological laboratories in regional centres and free them to perform the more time consuming therapeutic interventions such as radiofrequency ablation.

1 Jackman WM, Beckman $\mathrm{KJ}$, McClelland JH, Wong $\mathrm{X}$, Friday KJ, Roman CA. Treatment of supraventricular tachycardia due to atrioventricular nodal re-entry by radiofrequency catheter ablation of slow pathway con-
duction. $N$ Engl 9 Med 1992;327:313-8. Lee MA, Morday F, Kadish A, et al. Catheter modification of the atrioventricular junction with radiofrequency current for control of atrioventricular nodal re-entry current for control of atrioventricular

3 Jackman WM, Wang X, Friday KJ, et al. Catheter ablation of accessory atrioventricular pathways by radiofrequency current. N Engl $\Im$ Med 1991;324:1605-11. 
4 Waller TJ, Kay HR, Spielman SR, Kikalek SP, Greenspam AM, Horowitz LN. Reductions in sudden death and total mortality by antiarrhythmic therapy evaluation by electrophysiological drug testing: criteria of efficacy in patients with sustained ventricular tachyarrhythmias. f Am Coll Cardiol 1987;10:83-9.

5 Steinbeck G, Andersen D, Bach P, et al. A comparison of electrophysiology guided anti-arrhythmic drug therapy with beta-blocker therapy in patients with symptomatic sustained ventricular tachyarrhythmias. $N \mathrm{Engl} F \mathrm{Med}$ 1992;327:987-92.

6 Bauernfeind RA, Wyndham CR, Dhingra RC, et al. Serial electrophysiological testing of multiple drugs in patients with atrioventricular nodal re-entrant paroxysmal tachywith atrioventricular nodal re-entrat
cardia. Circulation 1980;62:1341.

7 Prystowsky EN. Indications for intracardiac electrophysiological studies in patients with supraventricular tachycardia. Circulation 1987;75 (suppl 3):119-22.
8 Ward DE, Camm AJ. Clinical electrophysiology of the heart. London: Arnold, 1982;277.

Horowitz LN. Safety of electrophysiological studies. Circulation 1986;73(suppl II):II-28.

10 Ward DE, Camm AJ. Clinical electrophysiology of the heart. London: Arnold 1982;16.

11 Ward DE, Camm AJ. Clinical electrophysiology of the heart London: Arnold, 1987;240.

12 Click RL, Gersh BJ, Sugrue DD, et al. Role of invasive electrophysiologic testing in patients with symptomatic electrophysiologic testing in patients with symp
bundle branch block. Am $₹$ Cardiol 1987;59:817.

13 Saksena S. Electrophysiological evaluation for recurrent tachycardias: a financial endangered technique. Int $\mathfrak{f}$ Cardiol 1985; $7: 431-6$

14 Ferguson D, Saksena S, Greenberg E, et al. Management of recurrent ventricular tachycardia: economic impact of therapeutic alternatives. Am $\mathcal{F}$ Cardiol 1984;53: 